\title{
The relationship between depression and risk of metabolic syndrome: a meta-analysis of observational studies
}

\author{
Yousef Moradi ${ }^{1}$, Ahmed N Albatineh ${ }^{2}$, Hassan Mahmoodi ${ }^{1}$ and Reza Ghanei Gheshlagh ${ }^{3 *}$
}

\begin{abstract}
Introduction: The link between metabolic syndrome and depression has always been controversial. Different studies that have examined the relationship between metabolic syndrome and depression have reported different results. Therefore, the goal of the present study was to examine the association between depression and MetS by meta-analysis.

Methods: Embase, Scopus, PubMed, and ISI were searched for publications in English from January 1990 to February 2020. Search included cohort and cross-sectional studies aimed at examining the association between depression and MetS. The risk of bias was assessed by Newcastle-Ottawa Scale. Heterogeneity and publication bias were tested, subgroup analysis and meta-regression were conducted.

Results: 49 studies with total sample size 399,494 were analyzed. Results indicated the odds of MetS was higher in depressed compared to non-depressed individuals [OR: 1.48; $95 \% \mathrm{Cl}$ : 1.33-1.64) vs. (OR: 1.38; $95 \% \mathrm{Cl}: 1.17-1.64)]$. For cross-sectional studies, depressed patients in Europe $(\mathrm{OR}=1.35 ; 95 \% \mathrm{Cl}$ : 1.47-1.99) were at higher odds of MetS compared to those in America and Asia. For cohort studies, depressed patients in America (OR=1.46; $95 \% \mathrm{Cl}$ : 1.161.84) were at higher odds of MetS than those in Europe. Cross-sectional studies indicated women with depression were at higher odds of MetS ( $\mathrm{OR}=1.95 ; 95 \% \mathrm{Cl}: 1.38-2.74)$ compared to men. In both types of studies, the odds of MetS decreased with age.
\end{abstract}

Conclusions: Metabolic syndrome is more common in depressed compared to non-depressed individuals.

Keywords: Depression, Metabolic syndrome, Observational study, Meta-analysis

\section{Introduction}

Metabolic syndrome (MetS) is a cluster of conditions, such as increase in waist circumference, dyslipidemia (elevated triglyceride levels and reduced HDL), increased blood pressure, and increased fasting blood sugar levels that is related to insulin resistance, diabetes, and elevated risk of cardiovascular disease $[1,2]$. MetS and

\footnotetext{
* Correspondence: rezaghanei30@yahoo.com

${ }^{3}$ Spiritual Health Research Center, Research Institute for Health Development, Kurdistan University of Medical Sciences, Sanandaj, Iran

Full list of author information is available at the end of the article
}

related metabolic biomarkers may be related to mood disorders, including depression [3].

Sedentary lifestyle and poor diet are known as the main causes of MetS that may be more common in depressed individuals compared to non-depressed people [4]. The high co-occurrence between MetS and mood disorders indicates high pathophysiological overlap between the two conditions [3]. The global prevalence of depression is increasing, and it is projected to become the second-leading cause of death by $2030[5,6]$.

There are hypothetical mechanisms by which there may be a link between MetS and depression. Due to low

C C The Author(s). 2021 Open Access This article is licensed under a Creative Commons Attribution 4.0 International License, which permits use, sharing, adaptation, distribution and reproduction in any medium or format, as long as you give appropriate credit to the original author(s) and the source, provide a link to the Creative Commons licence, and indicate if changes were made. The images or other third party material in this article are included in the article's Creative Commons licence, unless indicated otherwise in a credit line to the material. If material is not included in the article's Creative Commons licence and your intended use is not permitted by statutory regulation or exceeds the permitted use, you will need to obtain permission directly from the copyright holder. To view a copy of this licence, visit http://creativecommons.org/licenses/by/4.0/ The Creative Commons Public Domain Dedication waiver (http://creativecommons.org/publicdomain/zero/1.0/) applies to the data made available in this article, unless otherwise stated in a credit line to the data. 
physical activity, patients with depression are vulnerable to weight gain, MetS, and finally diabetes and cardiovascular diseases [7]. Previous studies on the association between MetS and depression have led to conflicting results. Some studies have found no significant association between the two conditions [8-12]; while others have found significant associations [13]. In light of the existing controversies about the relationship between these two variables, we felt that the literature on this subject needed to be re-evaluated. Therefore, the present systematic review and meta-analysis aims to provide pooled estimate of the association between MetS and depression which will offer an evidence-based answer to the association between depression and MetS can guide clinical management and therapy in decision making [14].

\section{Methods}

The present systematic review and meta-analysis was conducted according to the Preferred Reporting Items for Systematic Reviews and Meta-Analyses (PRISMA) according to the following 5 steps: Search Strategy and Search Terms, Eligibility Criteria and Study selection, Data Extraction, Quality Assessment and Risk of Bias, and Meta- analysis [15].

\section{Search strategy and search terms}

All internal databases, such as PsycInfo, Cochrane, Web of Sciences, Scopus, and Pub Med (Medline) were searched for articles published from January 1990 to February 2020. Grey literature (conference papers, related magazines etc.) was also examined. The search was conducted using the following keywords that were selected using Mesh and Emtree:

"Syndrome X", "Metabolic Syndrome", "Insulin Resistance Syndrome", "MetSyn", "Depression", "Depressive Disorder", "Dysthymic disorder". The search process was conducted by two independent researchers (RGH and $\mathrm{YM}$ ); any disagreement between them was resolved through discussion.

\section{Eligibility criteria and study selection}

At the end of the search process, all selected articles were included in EndNote, version 8. First, duplicate articles were identified then screened based on titles and abstracts. Second, full texts were examined, and the final articles were selected. The inclusion criteria were as follows: original observational cohort or cross-sectional studies, focused on depression as exposure, risk of MetS as outcome, and published in English. Case or casereport studies, letters to the editor, series, randomized clinical trials, studies not reporting the risk of MetS in depressed patients using the indices of effect size, such as odds ratio and risk ratio, articles without available fill texts, and articles published in languages other than English were excluded.

\section{Data extraction}

In the first step, titles and abstracts and in the next step, full texts were reviewed to extract the required information, such as name of the first author, year and country of publication, depression screening tools, diagnostic criteria of MetS, mean age of participants, number of patients with depression, number of patients with MetS. List of references for articles were also examined to identify other potential articles.

\section{Quality assessment and risk of bias}

The methodological quality of articles was examined by two independent researchers (RGH and YM) based on the 10 items in STROBE checklist. The 10 items assessed: title and abstract, objectives and hypotheses, inclusion criteria, sample size, statistical methods, descriptive data, interpretation of results, study limitations, and funding [16]. Higher scores on this checklist indicated better methodological quality. According to this score, articles were divided into three categories of methodological quality, including poor (4 or below), average [4-7] and good (over 7). Risk of bias was assessed using the Newcastle-Ottawa Quality Assessment Scale (NOS) [17]. The NOS assesses any study using 6 items in 3 groups, including selection, exposure, and comparability. The maximum score on the NOS is 9. When there are differences in the scores given to the selected articles, this is resolved through external discussion.

\section{Statistical analysis}

In the cross-sectional studies, the effect size of depression on MetS was reported using qualitative dichotomous (Yes-No) scores together with the odds ratio (OR). Logarithm and standard error of OR were used to combine the results of studies. ORs were combined with standard error of ORs using the random effects model. In cohort studies, the effect size of depression on MetS was reported using qualitative dichotomous (Yes-No) scores together with the risk ratio (RR); RRs were combined with standard error of RRs using the random effects model. For studies in which results were reported as percentages, first, the variance and standard error of each study were calculated using the binomial distribution, and then, the random or fixed effects model was used to combine the results of different studies. Heterogeneity among the studies was examined using the Cochran's Q test and the DerSimonian and Laird's statistical method. Cochran $\mathrm{Q}$ and I square $\left(\mathrm{I}^{2}\right)$ tests were used to investigate the heterogeneity and variance between studies. The Q statistic tells us whether there is 
statistically significant heterogeneity among the studies. The $\mathrm{I}^{2}$ value indicates the amount of heterogeneity quantitatively in a range from $0-100 \%$. Thresholds for the interpretation of $\mathrm{I}^{2}$ can be misleading, since the importance of inconsistency depends on several factors. A rough guide to interpretation is $0-40 \%$ (might not be important), 30-60\% (may represent moderate heterogeneity), 50-90\% (may represent substantial heterogeneity), and 75-100\% (considerable heterogeneity) [18]. In case of heterogeneity among studies, the random effects model was used to combine the results [19]. A funnel diagram, Egger's test, and its graphs were used to evaluate the publication bias. In the Egger's regression model, the ratio of the effect size on the standard error, which is the standard index (z-score), is taken as the dependent variable and predicts its value over the inverse of standard error (1/SE) [19]. Forest plot was produced, and meta-regression was conducted to examine the association between sample size, year of publication, and mean age of participants with odds of MetS. Additionally, subgroup analysis was conducted by gender (women, men, and both), depression screening tool, diagnostic criteria of MetS (WHO, IDF, ATP III), population (patients, general population), mean age of patients (below or over 50 years), and geographical location (Asia and Australia, Europe and America). Some studies reported their results by gender or instrument rather than reporting total prevalence rates; for these studies, two groups were included in the analysis. The data was analyzed using Stata, version 16.

\section{Results}

In the initial search, 2996 non-duplicate articles were identified that were potentially eligible. In the screening process, 146 articles were maintained after excluding the unrelated ones. In the next step, the eligibility of articles was examined; of which 102 articles were excluded, and 31 articles were included in the final analysis. Table 1 shows the process of screening and selecting the articles. (Fig. 1)

\section{Study and participants' characteristics}

Overall, 49 articles (31 cross-sectional studies in 36 groups and 18 cohort studies in 21 groups) were included in the analysis. The total sample sizes of the cross-sectional and cohort studies were 111,866 and 287, 628 , respectively. Most of cross-sectional studies (4 studies in each country) had been conducted in Brazil [3, 20-22] and South Korea [23-26]; and most cohort studies $(n=6)$ had been conducted in America [7, 27-31].

Among cross-sectional studies, the highest and lowest sample sizes were for the studies by Park [26] $(n=23$, $385)$ and Chattopadhyay [32] $(n=97)$, respectively; and in four studies, the sample included over 10,000 participants [11, 24, 26, 33]. Among cohort studies, the highest and lowest sample sizes were for the studies by Dregan [34] $(n=124,445)$ and Viinamaki [35] $(n=223)$, respectively. Results were reported by gender in four studies $[13,25,36,37]$ and by type of depression (typical vs. atypical) in one study [38]; these studies were included in the analysis as two separate studies. In addition, three cross-sectional studies reported their results by gender; these were included in the analysis as three separate studies [7, 34, 39]. One cross-sectional study was focused on nursing personnel [22], and one on office workers [1]. Participants' type of illness was clearly stated only in two studies, one among patients with mental disorders [40] and one among patients with type II diabetes [41]. Among 21 cohort studies, 19 were among the general population, one was among patients with cardiovascular disease [31], and one was among office staff [42]. Further details are provided in Table 1.

\section{Meta-analysis of cross-sectional studies Depression and risk of metabolic syndrome}

Overall, the results showed that the pooled OR of MetS in patients with depression was $1.48 \%$ (95\% CI: $1.33-$ 1.64). Heterogeneity was found to be $\mathrm{I}^{2}=52.43 \%$, and the Cochran's $Q$ test led to a statistically significant result $(\mathrm{Q}=67.26, \mathrm{DoF}=32, p=0.003$ ) (Fig. 2).

Also, the meta-regression indicated that the log odds of MetS in patients with depression significantly decreased with age (estimated $\beta$ : -0.017 , SE: $0.007, p=$ $0.021,95 \%$ CI $-0.033,-0.002)$. This means for every year increase in age, there is $1.7 \%$ decrease in the odds of developing MetS in patients with depression (OR = $0.983,95 \%$ CI: $0.968,0.998$ ) and so older age has a protective effect against MetS in depressed patients. In addition, the funnel plot and the results of the Egger's test (Coefficient: 0.09, SE: 0.394, $p=0.794$ ) indicated no publication bias exists in the studies $(p=0.590)$ (Fig. 3).

\section{Subgroup analysis}

Results of subgroup analysis by geographical location showed that the pooled ORs of the studies conducted in Europe $(\mathrm{OR}=1.71 ; 95 \% \mathrm{CI}: 1.47-1.99)$ were higher compared to the studies conducted in America (OR = 1.45; $95 \%$ CI: $0.94-2.25)$ and Asia $(\mathrm{OR}=1.37$; $95 \% \mathrm{CI}$ : $1.26-1.49)$. Heterogeneity was only significant for studies conducted in America $\left(\mathrm{I}^{2}=79.28 \%, p=0.010\right)$. Results by gender indicated that depressed men were more likely to develop MetS compared to depressed women [(OR = 1.52; 95 \% CI: $1.13-2.05)$ vs. (OR $=1.35$; 95 \% CI: 0.90 $2.01)$, though women pooled estimate is not significant. 
Table 1 Main characteristics of the selected studies

\begin{tabular}{|c|c|c|c|c|c|c|c|c|c|}
\hline \multirow[t]{2}{*}{ Study Type } & \multirow[t]{2}{*}{ First Author } & \multirow[t]{2}{*}{ Year } & \multirow{2}{*}{$\begin{array}{l}\text { Sample } \\
\text { size }\end{array}$} & \multirow[t]{2}{*}{ Age } & \multirow[t]{2}{*}{ Country } & \multirow[t]{2}{*}{ Target } & \multicolumn{2}{|l|}{ Scale } & \multirow{2}{*}{$\begin{array}{l}\text { NOS } \\
\text { Score }\end{array}$} \\
\hline & & & & & & & Depression & MetS & \\
\hline \multirow[t]{36}{*}{ Cross-sectional studies } & Ko [23] & 2019 & 9867 & $\geq 19$ & Korea & General population & PHQ-9 & ATP III & 8 \\
\hline & Moreira [20] & 2019 & 545 & $18-24$ & Brazil & Young adults & Interview & ATP III & 6 \\
\hline & Chattopadhyay [32] & 2018 & 97 & - & India & Primary care patients & BDI & ATP III & 7 \\
\hline & Bakhtiari [43] & 2018 & 1560 & $69.3 \pm 7.4$ & Iran & Elderly people & GSD & ATP III & 7 \\
\hline & Mattei [44] & 2018 & 129 & $40-80$ & Italy & Primary care patients & HADS & ATP III & 7 \\
\hline & Kim [24] & 2018 & 10,459 & - & Korea & General population & PHQ-9 & ATP III & 7 \\
\hline & Moreira [21] & 2017 & 972 & $25.8 \pm 2.1$ & Brazil & General population & Interview & ATP III & 7 \\
\hline & $\operatorname{Ra}[25]$ & 2017 & 1938 & $\geq 40$ & Korea & Men & Interview & ATP III & 7 \\
\hline & & & 2404 & & & Women & & & 7 \\
\hline & Yu [11] & 2017 & 11,430 & $\geq 35$ & China & General population & PHQ-9 & ATP III & 8 \\
\hline & Chang [12] & 2017 & 11,258 & - & Taiwan & General population & MHI-5 & WHO & 8 \\
\hline & Cardenas [45] & 2017 & 332 & $\geq 60$ & USA & Elderly & PHQ-9 & WHO & 8 \\
\hline & Park [26] & 2016 & 23,385 & $46.13 \pm 0.18$ & Korea & Women & Interview & ATP III & 8 \\
\hline & Agarwal [40] & 2016 & 150 & - & India & psychiatric Outpatient & Interview & ATP III & 7 \\
\hline & Ribeiro [22] & 2015 & 226 & $23-66$ & Brazil & Nursing personnel & HADS & ATP III & 7 \\
\hline & Kahl [46] & 2015 & 163 & - & Germany & Primary care patients & Interview & ATP III & 7 \\
\hline & Vargas [3] & 2014 & 342 & - & Brazil & General population & Interview & IDF & 7 \\
\hline & Butnoriene [47] & 2014 & 1115 & $62 \pm 9.6$ & Lithuania & General population & Interview & ATP III & 7 \\
\hline & Takeuchi [38] & 2013 & 1011 & $41.1 \pm 8.1$ & Japan & General population & Interview & IDF & 8 \\
\hline & & & 1011 & $42.3 \pm 8.7$ & & & & & 8 \\
\hline & Sekita [13] & 2013 & 1353 & 63 & Japan & Men & CES-D & ATP III & 7 \\
\hline & & 2013 & 1760 & 62 & & Women & & & 7 \\
\hline & Marijnissen & 2013 & 1277 & $61.1 \pm 5.9$ & Netherlands & General population & BDI-I & IDF & 7 \\
\hline & Demirci [48] & 2011 & 250 & - & Turkey & General population & BDI & ATP III & 7 \\
\hline & Foley [8] & 2010 & 2525 & - & Australia & General population & Interview & ATP III & 8 \\
\hline & Ahola [41] & 2010 & 1226 & $45 \pm 12$ & Finland & Diabetes type I & $\mathrm{BDI}$ & ATP III & 8 \\
\hline & Hildrum [9] & 2009 & 9571 & 47.74 & Norway & General population & HADS & IDF & 8 \\
\hline & Takeuchi [1] & 2009 & 1215 & 42.5 & Japan & Office workers & POMS & IDF & 8 \\
\hline & Toker [36] & 2008 & 1525 & $20-75$ & Israel & Women & PHQ-9 & ATP III & 7 \\
\hline & & & 2355 & $20-75$ & & Men & & & 7 \\
\hline & Miettola [49] & 2008 & 416 & $50.4 \pm 10.5$ & Finland & General population & $\mathrm{BDI}$ & ATP III & 6 \\
\hline & Dunbar [50] & 2008 & 1345 & $25-84$ & Australia & General population & HADS & ATP III & 7 \\
\hline & Vogelzangs [51] & 2007 & 867 & $74.1 \pm 6.6$ & Italy & General population & CES-D & ATP III & 7 \\
\hline & Skilton [52] & 2007 & 1598 & $51.8 \pm 9.8$ & France & Primary care patients & HADS & ATP III & 7 \\
\hline & Kinder [37] & 2004 & 3003 & 28.7 & USA & Women & Interview & ATP III & 7 \\
\hline & & & 3186 & 28.2 & & Men & & & 7 \\
\hline \multirow[t]{7}{*}{ Cohort studies } & Dregan [34] & 2020 & 71,799 & - & United Kingdom & Women & Interview & ATP III & 8 \\
\hline & & & 52,646 & - & & Men & & & 8 \\
\hline & Matta [4] & 2019 & 64,861 & 46.30 & France & General population & CES-D & IDF & 7 \\
\hline & van Leijden [2] & 2018 & 21,182 & $44.2 \pm 13.2$ & Netherlands & Multi-ethnic & PHQ-9 & IDF & 7 \\
\hline & Rethorst [27] & 2017 & 47,702 & - & USA & General population & Interview & ATP III & 7 \\
\hline & Marriana [53] & 2017 & 1172 & 62 & Finland & General population & CES-D & ATP III & 7 \\
\hline & Matthew [28] & 2016 & 1743 & 52.5 & USA & General population & CES-D & ATP III & 7 \\
\hline
\end{tabular}


Table 1 Main characteristics of the selected studies (Continued)

\begin{tabular}{|c|c|c|c|c|c|c|c|c|c|}
\hline \multirow[t]{2}{*}{ Study Type } & \multirow[t]{2}{*}{ First Author } & \multirow[t]{2}{*}{ Year } & \multirow{2}{*}{$\begin{array}{l}\text { Sample } \\
\text { size }\end{array}$} & \multirow[t]{2}{*}{ Age } & \multirow[t]{2}{*}{ Country } & \multirow[t]{2}{*}{ Target } & \multicolumn{2}{|l|}{ Scale } & \multirow{2}{*}{$\begin{array}{l}\text { NOS } \\
\text { Score }\end{array}$} \\
\hline & & & & & & & Depression & MetS & \\
\hline & Renel [29] & 2015 & 1798 & 43.3 & USA & General population & CES-D & ATP III & 7 \\
\hline & Akbaraly [54] & 2011 & 4446 & $\geq 65$ & France & Elderly & CES-D & ATP III & 7 \\
\hline & East [7] & 2010 & 1688 & $47.6 \pm 10.3$ & USA & Women & CES-D & ATP III & 7 \\
\hline & & & 3437 & $49.3 \pm 10.2$ & & Men & & & 7 \\
\hline & Akbaraly [42] & 2009 & 5232 & $49.5 \pm 6.1$ & France & Office staff & GHQ & ATP III & 8 \\
\hline & Goldbacher [30] & 2009 & 429 & 45.6 & USA & Women & DSM-IV & ATP III & 7 \\
\hline & Vogelzangs [10] & 2009 & 1212 & $55-85$ & Netherlands & General population & CES-D & ATP III & 8 \\
\hline & Vanhala [39] & 2009 & 294 & 45.9 & Finland & Women & $\mathrm{BDI}$ & ATP III & 6 \\
\hline & & & 194 & 46 & & Men & & & 6 \\
\hline & Viinamaki [35] & 2009 & 223 & - & Finland & General population & DSM-IV & ATP III & 6 \\
\hline & Vaccarino [31] & 2008 & 652 & - & USA & Cardiovascular disease & $\mathrm{BDI}$ & ATP III & 7 \\
\hline & Katri [55] & 2007 & 432 & 49 & Finland & General population & $\mathrm{BDI}$ & ATP III & 7 \\
\hline & Gil [56] & 2006 & 795 & - & Poland & General population & $\mathrm{BDI}$ & ATP III & 7 \\
\hline & Herva [57] & 2006 & 5691 & - & Finland & General population & HSCL-25 & ATP III & 8 \\
\hline
\end{tabular}

Abbreviations: BDI-I Beck Depression Inventory; CES-D Center for Epidemiologic Survey-Depression; DSM Diagnostic and Statistical Manual of Mental Disorders; GHQ General Health Questionnaire; GSD Geriatric Depression Scale Hopkins Symptom Checklist; HSCL Hopkins Symptom Checklist; IDF International Diabetes Federation; MHI-5 5-item Mental Health Inventory; MINI Mini International Neuropsychiatric Interview; NCEP-ATP III National Cholesterol Education Program-Adult Treatment Panel III; PHQ Patient Health Questionnaire; POMS Profile of mood states; WHO World Health Organization

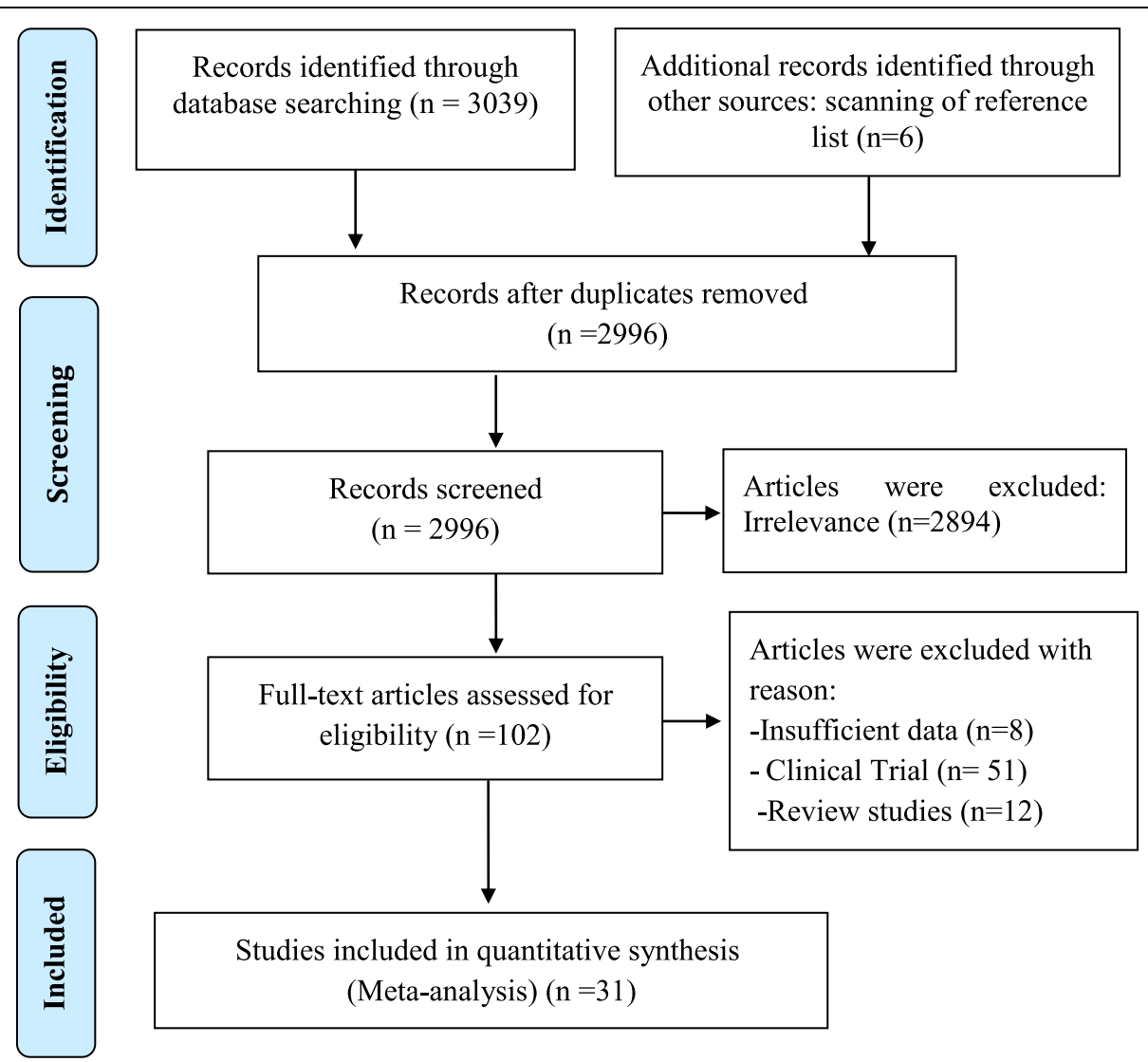

Fig. 1 Process of searching for, screening, and selecting the eligible articles 


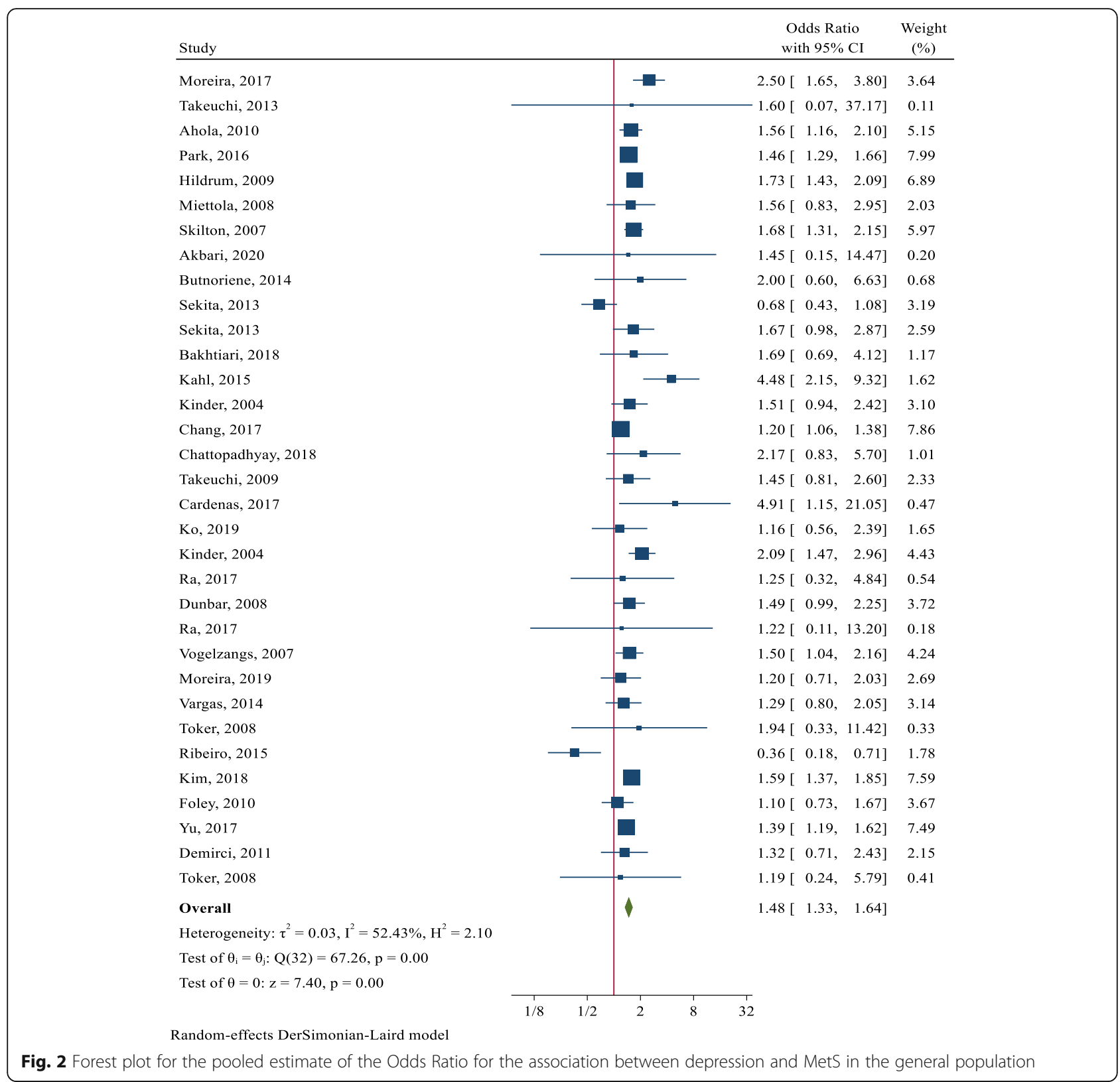

In addition, the results of subgroup analysis showed that pooled ORs were higher in the studies that used interview to examine depression ( $\mathrm{OR}=1.70$; $95 \% \mathrm{CI}$ : 1.37 2.12) (compared to questionnaires), and that the pooled ORs of the WHO's diagnostic criteria of MetS $(\mathrm{OR}=2.0$; $95 \% \mathrm{CI}: 0.53-7.52)$ were higher compared to those of the IDF (OR $=1.64 ; 95 \%$ CI: $1.39-1.94)$ and the ATP III $(\mathrm{OR}=1.48 ; 95 \%$ CI: 1.32-1.67) (Table 2).

\section{Meta-analysis of cohort studies}

Depression and risk of metabolic syndrome

Analysis of cohort studies showed that the pooled Risk Ratio of MetS in patients with depression was 1.38 (95\%
CI: 1.17-1.64). Heterogeneity was found to be $97.56 \%$, and the Cochran's $\mathrm{Q}$ test led to a statistically significant result $(\mathrm{Q}=818.20, \mathrm{DoF}=20, p<0.001)$ (Fig. 4).

\section{Subgroup analysis}

Results of subgroup analysis by continent showed that pooled ORs were higher in the studies conducted in America $(\mathrm{OR}=1.46 ; 95 \% \mathrm{CI}: 1.16-1.84)$ compared to those conducted in Europe $(\mathrm{OR}=1.28$; $95 \%$ CI: $0.95-$ 1.73). Heterogeneity was significant for the cohort studies conducted in America $\left(\mathrm{I}^{2}=98.51 \%\right)$ and Europe $\left(\mathrm{I}^{2}=92.41 \%\right)(\mathrm{p}<0.001)$. Results by gender showed that depressed men were more likely to develop MetS 


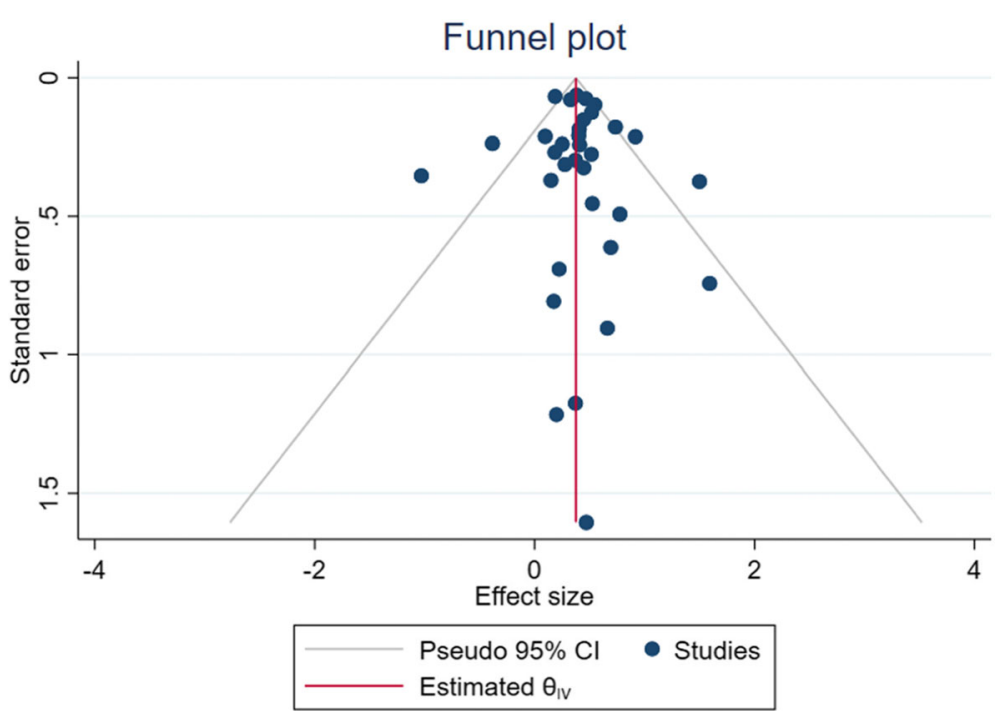

Fig. 3 Funnel plot for testing publication bias in the pooled estimate of the association between depression and MetS in the general population

compared to depressed women $[\mathrm{OR}=1.55 ; 95 \% \mathrm{CI}$ : $1.23-1.94)$ vs. $(\mathrm{OR}=1.54 ; 95 \% \mathrm{CI}: 1.11-2.14)]$. Additionally, participants under 50 years of age were more likely to develop MetS (OR $=1.30$; $95 \%$ CI: 1.13-149) compared to those over 50 years. Results of subgroup analysis also showed that pooled ORs were higher in the studies assessing depression using the $\mathrm{BDI}(\mathrm{OR}=1.50$; $95 \% \mathrm{CI}: 1.02-2.20)$ compared to those assessing this variable using other scales. Moreover, pooled ORs of the ATP III criteria (OR $=1.41 ; 95 \%$ CI: $1.18-1.69)$ for

Table 2 Summary of odds ratio estimates [95\% Cls] for cross-sectional studies focused on the association between depression and risk of MetS by gender, study population, continent, depression scales, MTs Scales, and Age

\begin{tabular}{|c|c|c|c|c|c|c|c|}
\hline \multirow[t]{2}{*}{ Subgroup } & \multirow{2}{*}{$\begin{array}{l}\text { Number of } \\
\text { studies }\end{array}$} & \multirow{2}{*}{$\begin{array}{l}\text { Summary OR } \\
(95 \% \mathrm{Cl})\end{array}$} & \multicolumn{3}{|c|}{ Between studies } & \multicolumn{2}{|c|}{ Between subgroups } \\
\hline & & & $\mathrm{I}^{2}$ & $\mathbf{P}_{\text {heterogeneity }}$ & $\mathbf{Q}$ & $\bar{Q}$ & $P_{\text {heterogeneity }}$ \\
\hline $\begin{array}{l}\text { Gender } \\
\text { Female } \\
\text { Male } \\
\text { Both }\end{array}$ & $\begin{array}{l}5 \\
5 \\
22\end{array}$ & $\begin{array}{l}1.35(0.90-2.01) \\
1.52(1.13-2.05) \\
1.49(1.31-1.69)\end{array}$ & $\begin{array}{l}70.37 \% \\
0.00 \% \\
59.94 \%\end{array}$ & $\begin{array}{l}0.01 \\
0.99 \\
0.01\end{array}$ & $\begin{array}{l}14.48 \\
0.27 \\
52.42\end{array}$ & 0.26 & 0.88 \\
\hline $\begin{array}{l}\text { Continents } \\
\text { America } \\
\text { Asia \& Australia } \\
\text { Europe }\end{array}$ & $\begin{array}{c}6 \\
16 \\
9\end{array}$ & $\begin{array}{l}1.45(0.94-2.25) \\
1.37(1.26-1.49) \\
1.71(1.47-1.99)\end{array}$ & $\begin{array}{l}79.28 \% \\
13.03 \% \\
21.69 \%\end{array}$ & $\begin{array}{l}0.01 \\
0.30 \\
0.26\end{array}$ & $\begin{array}{l}28.96 \\
20.70 \\
7.66\end{array}$ & 6.38 & 0.04 \\
\hline $\begin{array}{l}\text { Depression Scales } \\
\text { BDI } \\
\text { CES-D } \\
\text { Interview \& DSM } \\
\text { PHQ-9 } \\
\text { Others }\end{array}$ & $\begin{array}{c}5 \\
2 \\
11 \\
5 \\
8\end{array}$ & $\begin{array}{l}1.52(1.19-1.94) \\
1.20(0.70-2.05) \\
1.70(1.37-2.12) \\
1.49(1.34-1.66) \\
1.36(1.09-1.69)\end{array}$ & $\begin{array}{l}0.00 \% \\
76.72 \% \\
50.54 \% \\
67.90 \% \\
0.00 \%\end{array}$ & $\begin{array}{l}0.88 \\
0.01 \\
0.03 \\
<0.001 \\
0.44\end{array}$ & $\begin{array}{l}0.25 \\
8.59 \\
20.22 \\
28.03 \\
4.77\end{array}$ & 2.76 & 0.60 \\
\hline $\begin{array}{l}\text { MTs Scales } \\
\text { ATP III } \\
\text { IDF } \\
\text { WHO }\end{array}$ & $\begin{array}{l}27 \\
4 \\
2\end{array}$ & $\begin{array}{l}1.48(1.32-1.67) \\
1.64(1.39-1.94) \\
2.00(0.53-7.52)\end{array}$ & $\begin{array}{l}50.56 \% \\
0.00 \% \\
71.85 \%\end{array}$ & $\begin{array}{l}<0.001 \\
0.68 \\
0.06\end{array}$ & $\begin{array}{l}52.69 \\
1.51 \\
3.55\end{array}$ & 1.09 & 0.58 \\
\hline $\begin{array}{l}\text { Study Population } \\
\text { General Population } \\
\text { Patients } \\
\text { Other }\end{array}$ & $\begin{array}{l}27 \\
4 \\
2\end{array}$ & $\begin{array}{l}1.46(1.33-1.60) \\
1.95(1.38-2.74) \\
0.73(0.18-2.88)\end{array}$ & $\begin{array}{l}35.25 \% \\
58.25 \% \\
89.16 \%\end{array}$ & $\begin{array}{l}0.04 \\
0.07 \\
<0.001\end{array}$ & $\begin{array}{l}40.15 \\
7.19 \\
9.22\end{array}$ & 3.54 & 0.17 \\
\hline $\begin{array}{l}\text { Age } \\
\leq 50 \text { Year } \\
>50 \text { Year }\end{array}$ & $\begin{array}{l}5 \\
7\end{array}$ & $\begin{array}{l}1.66(1.41-1.94) \\
1.39(1.00-1.95)\end{array}$ & $\begin{array}{l}42.62 \% \\
50.97 \%\end{array}$ & $\begin{array}{l}0.14 \\
0.06\end{array}$ & $\begin{array}{l}6.97 \\
12.24\end{array}$ & 0.82 & 0.36 \\
\hline
\end{tabular}

Abbreviations: BDI-I Beck Depression Inventory; CES-D Center for Epidemiologic Survey-Depression; DSM Diagnostic and Statistical Manual of Mental Disorders; IDF International Diabetes Federation; MHI-5 5-item Mental Health Inventory; NCEP-ATP III National Cholesterol Education Program-Adult Treatment Panel III; PHQ Patient Health Questionnaire; WHO World Health Organization 


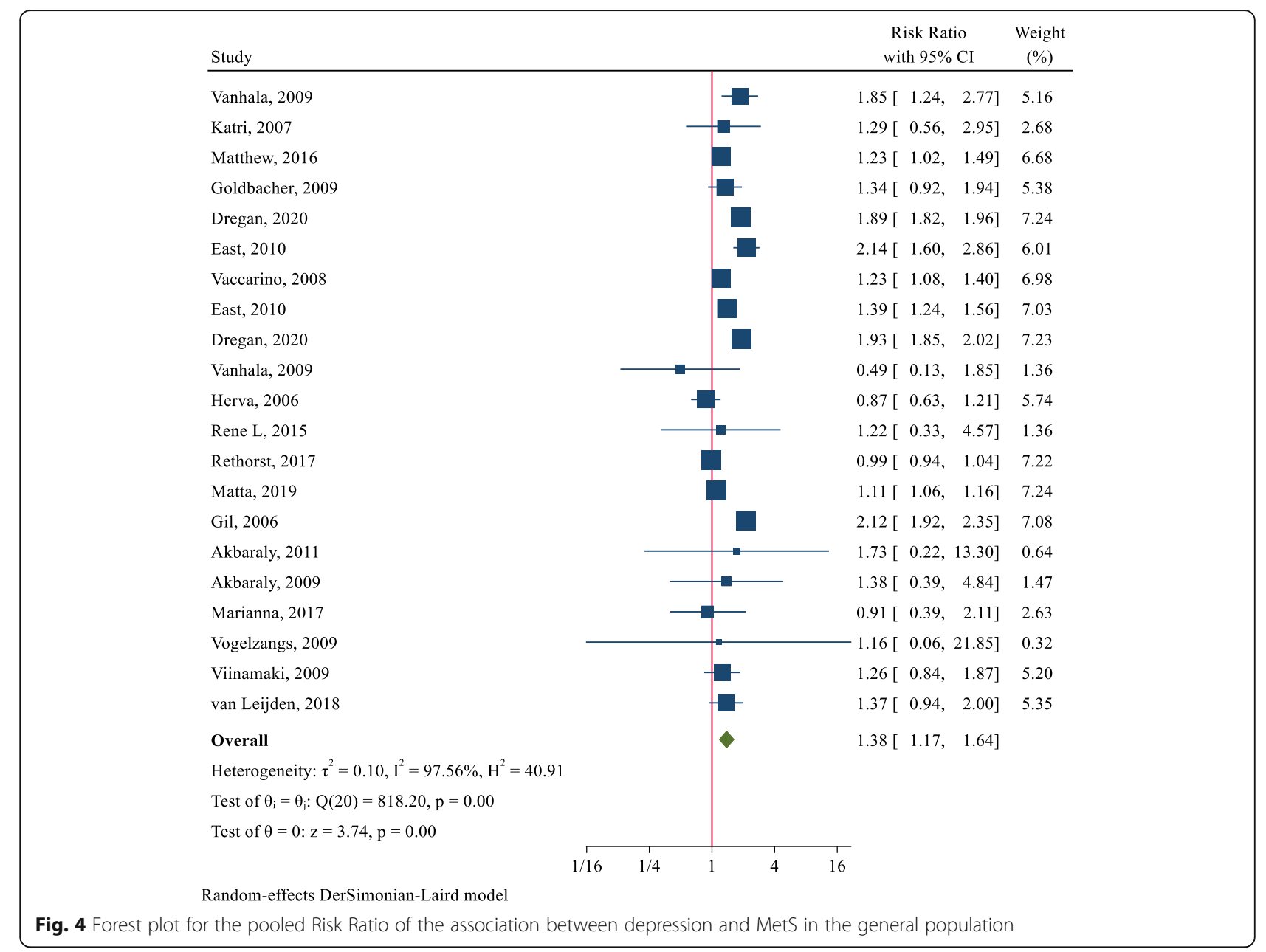

diagnosis of MetS were higher compared to those of the IDF criteria (OR = 1.13; 95 \% CI: 1.01-1.27) (Table 3).

Also, meta-regression analysis indicated that the $\log \beta$ (risk ratio) of MetS in patients with depression is not associated with age $(\beta:-0.011$, SE: $0.024, p=0.637,95 \%$ CI: $-0.059,-0.036)$. Additionally, the funnel plot and the results of Egger test (Coefficient: -0.44, SE: 0.449, $p=0.331)$ indicated no publication bias in the studies $(p=0.437)$ (Fig. 5).

\section{Discussion}

Our meta-analysis of 31 cross-sectional and 18 cohort studies indicted a significant relationship between depression and MetS. The results of a previous metaanalysis in which 16 articles were analyzed showed that there was a relationship between metabolic syndrome and depression. The present meta-analyses showed that depressed patients were more likely to have MetS compared to non-depressed patients [58-60]. In the previous meta-analysis [58], 16 articles were reviewed, but the articles analyzed in this study had increased to 49 cases. The increase in the number of articles in this field indicates the interest of researchers and it seemed necessary to re-examine the controversial relationship between these variables.

Although the mechanism of this association is unknown, some hypotheses have been suggested. Depression can lead to MetS through different mechanisms. Firstly, depressed patients tend to have adverse health behaviors, such as alcohol consumption, cigarette smoking, poor diet, and may have a sedentary lifestyle that all can have a role that leads to MetS $[30,58]$. Secondly, depressed patients are less likely to follow their treatment regimen [61]. Results of a meta-analysis by DiMatteo et al. (2000) showed that depressed patients were twice as less likely to adhere to treatment than non-depressed individuals [62]. Thirdly, antidepressants may lead to MetS through increasing the risk of abdominal obesity, high blood pressure, and increased triglycerides levels [63]. Akbaraly indicated that there is a mutual association between depression and MetS; he calls this association a "two-way street" [54]. Theoretically, depression 
Table 3 Summary of relative risk estimates (RR) (95\% Cls) for cohort studies that assess the association between depression and risk of MetS by gender, study population, continent, depression scales, MetS scales, and Age.

\begin{tabular}{|c|c|c|c|c|c|c|c|}
\hline \multirow[t]{2}{*}{ Subgroup } & \multirow{2}{*}{$\begin{array}{l}\text { Number } \\
\text { of } \\
\text { studies }\end{array}$} & \multirow{2}{*}{$\begin{array}{l}\text { Summary } \\
\text { Relative Risk } \\
(95 \% \mathrm{CI})\end{array}$} & \multicolumn{3}{|c|}{ Between studies } & \multicolumn{2}{|c|}{ Between subgroups } \\
\hline & & & $\overline{1^{2}}$ & $P_{\text {heterogeneity }}$ & $\mathbf{Q}$ & $\overline{\mathrm{Q}}$ & $P_{\text {heterogeneity }}$ \\
\hline $\begin{array}{l}\text { Gender } \\
\text { Female } \\
\text { Male } \\
\text { Both }\end{array}$ & $\begin{array}{l}7 \\
3 \\
11\end{array}$ & $\begin{array}{l}1.55(1.23-1.94) \\
1.54(1.11-2.14) \\
1.23(0.99-1.53)\end{array}$ & $\begin{array}{l}89.04 \% \\
93.61 \% \\
94.47 \%\end{array}$ & $\begin{array}{l}<0.001 \\
<0.001 \\
<0.001\end{array}$ & $\begin{array}{l}59.64 \\
31.28 \\
89.61\end{array}$ & 2.40 & 0.30 \\
\hline $\begin{array}{l}\text { Continents } \\
\text { America } \\
\text { Europe }\end{array}$ & $\begin{array}{c}9 \\
12\end{array}$ & $\begin{array}{l}1.46(1.16-1.84) \\
1.28(0.95-1.73)\end{array}$ & $\begin{array}{l}98.51 \% \\
92.41 \%\end{array}$ & $\begin{array}{l}<0.001 \\
<0.001\end{array}$ & 99.7995 .84 & 0.45 & 0.50 \\
\hline $\begin{array}{l}\text { Depression Scales } \\
\text { BDI } \\
\text { CES-D } \\
\text { Interview\& DM } \\
\text { Other }\end{array}$ & $\begin{array}{l}5 \\
8 \\
5 \\
3\end{array}$ & $\begin{array}{l}1.50(1.02-2.20) \\
1.34(1.11-1.61) \\
1.45(1.05-2.00) \\
1.10(0.77-1.56)\end{array}$ & $\begin{array}{l}91.39 \% \\
77.66 \% \\
99.21 \% \\
38.96 \%\end{array}$ & $\begin{array}{l}<0.001 \\
<0.001 \\
<0.001 \\
0.19\end{array}$ & $\begin{array}{c}46.48 \\
31.34 \\
98.27 \\
3.28\end{array}$ & 1.81 & 0.61 \\
\hline $\begin{array}{l}\text { MetS Scales } \\
\text { ATP III } \\
\text { IDF }\end{array}$ & $\begin{array}{l}19 \\
2\end{array}$ & $\begin{array}{l}1.41(1.18-1.69) \\
1.13(1.01-1.27)\end{array}$ & $\begin{array}{l}96.94 \% \\
14.80 \%\end{array}$ & $\begin{array}{l}<0.001 \\
0.28\end{array}$ & $\begin{array}{r}588.0 \\
1.17\end{array}$ & 4.14 & 0.04 \\
\hline $\begin{array}{l}\text { Study Population } \\
\text { General } \\
\text { Patients } \\
\text { Other }\end{array}$ & $\begin{array}{l}19 \\
1 \\
1\end{array}$ & $\begin{array}{l}1.46(1.33-1.60) \\
- \\
-\end{array}$ & $\begin{array}{l}97.78 \% \\
- \\
-\end{array}$ & $\begin{array}{l}<0.001 \\
- \\
-\end{array}$ & $\begin{array}{r}810.7 \\
- \\
-\end{array}$ & 1.32 & 0.52 \\
\hline $\begin{array}{l}\text { Age } \\
\leq 50 \text { Year } \\
>50 \text { Year }\end{array}$ & $\begin{array}{l}11 \\
2\end{array}$ & $\begin{array}{l}1.30(1.13-1.49) \\
1.21(1.13-1.44)\end{array}$ & $\begin{array}{l}84.26 \% \\
0.00 \%\end{array}$ & $\begin{array}{l}<0.001 \\
0.49\end{array}$ & $\begin{array}{l}63.54 \\
0.47\end{array}$ & 0.34 & 0.59 \\
\hline
\end{tabular}

can activate the hypothalamic-pituitary-adrenal axis and lead to accumulation of visceral fat through increasing the secretion of corticotrophin-releasing hormone, adrenocorticotropic hormone, and cortisol [50].

Moreover, results of cross-sectional studies indicated that the pooled ORs of MetS were higher in depressed patients than in the general population, and higher in America than in Asia. Also, in the included cohort studies, the risk of MetS in depressed patients with underlying conditions was higher than in the General population and higher in America compared to Europe. This finding can be attributed to the type of study methodology and the characteristics of the studied samples. Various studies have shown an association between depression and diet [64-66]. Le Port et al. indicated that fruits and fish diet was related to lower risk of depression symptoms [67]. Huang et al. (2019) have also shown that a healthy diet such as a Mediterranean diet and

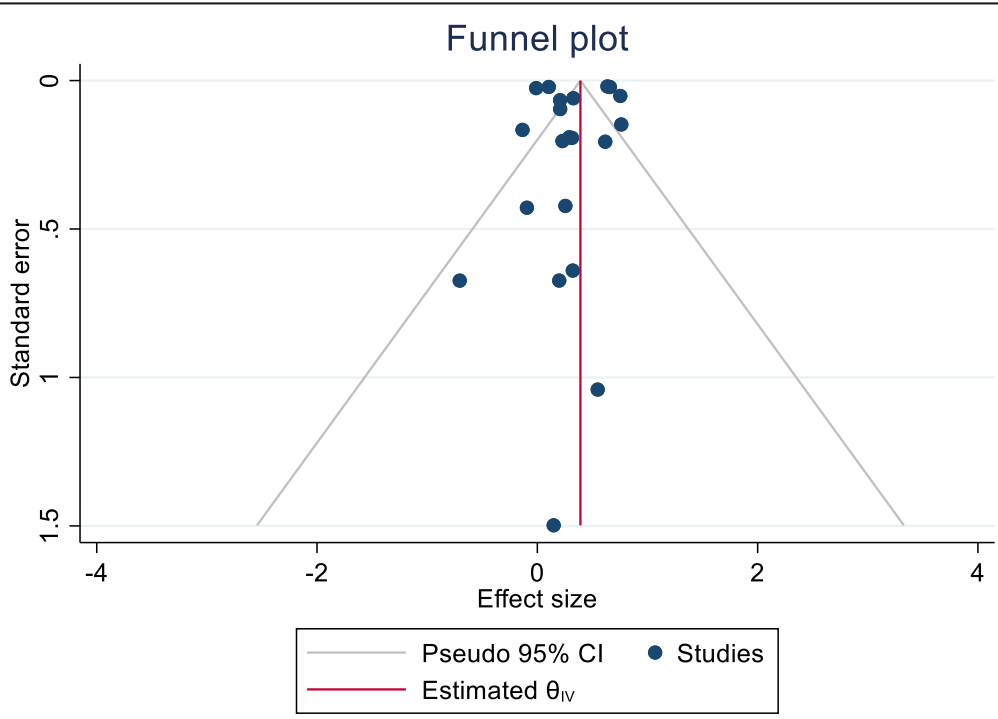

Fig. 5 Funnel plot for testing for publication bias in the pooled estimate of the effect of depression on the risk of MetS in the general population 
certain foods such as fish, fresh vegetables, and fruits can reduce depression [68]. Also, Allison stated that in populations with different sociocultural backgrounds, there are different risk factors as a result of different genetic and socioeconomic factors [69].

Results of the included cross-sectional studies by gender indicated that pooled ORs were higher in studies conducted in men compared to those conducted in women or mixed group of men and women. Results of cohort studies showed that the risk of MetS was almost the same in men and women. Results of some studies were influenced by gender; for example, in some studies, the association between depression and MetS was only significant in men $[13,56]$, while in some others, this association was only significant in women $[30,37,55,70]$. This finding can be explained by the fact that compared to women, men are more likely to have an unhealthy lifestyle, such as drinking alcohol or eating fast food, tend to pay less attention to their appearance and weight, and may be less willing to go to the doctor when experiencing physical problems [71, 72].

The results of cross-sectional studies showed that pooled ORs of MetS in depressed patients were higher in studies assessing depression using interviews and assessing MetS using the WHO's criteria compared to studies assessing these two conditions using other tools or criteria. The cohort studies that used the BDI and the ATP III to screen for depression and MetS reported a higher risk of MetS in depressed patients compared to those that used other tools or criteria. Only two crosssectional studies used the WHO criteria; this may have influenced the generalizability of their results. Most of the cohort studies used the ATP III criteria to diagnose MetS which is more common than the other criteria. According the regression analysis of cross-sectional and cohort studies, the risk of MetS in depressed patients decreased with age; further studies are needed to explain this finding.

Some strengths of the present study includes the focus on a new topic, extra study details, large number of studies, and large number of patients included in the meta-analysis.

\section{Conclusions}

The results of the present systematic review and metaanalysis indicated a relationship between depression and metabolic syndrome (MetS). Understanding this association is important because a history of depression, which predicts the risk of cardiovascular disease in the future, that can worsen underlying health conditions and may increase mortality rates.

\section{Acknowledgements}

The authors appreciate all the researchers whose articles were used in the present research.
Authors' contributions

RGG and HM: data collection; RGG and YM: study design; ANA: final revision and grammar editing; YM: statistical analysis. all authors have read and approved the manuscript.

\section{Funding}

None.

Availability of data and materials

The datasets used and/or analyzed during the current study are available from the corresponding author on reasonable request.

Ethics approval and consent to participate

Not applicable.

Consent for publication

Not applicable.

\section{Competing interests}

The authors declare that they have no competing interests.

\section{Author details}

'Social Determinants of Health Research Center, Research Institute for Health Development, Kurdistan University of Medical Sciences, Sanandaj, Iran.

${ }^{2}$ Department of Community Medicine and Behavioral Sciences, Faculty of Medicine, Kuwait University, Kuwait City, Kuwait. ${ }^{3}$ Spiritual Health Research Center, Research Institute for Health Development, Kurdistan University of Medical Sciences, Sanandaj, Iran.

Received: 8 August 2020 Accepted: 5 January 2021

Published online: 02 March 2021

\section{References}

1. Takeuchi T, Nakao M, Nomura K, Yano E. Association of metabolic syndrome with depression and anxiety in Japanese men. Diabetes Metab. 2009;35(1): 32-6.

2. van Leijden MJ, Penninx BW, Agyemang C, Olff M, Adriaanse MC, Snijder $M B$. The association of depression and posttraumatic stress disorder with the metabolic syndrome in a multi-ethnic cohort: the HELIUS study. Soc Psychiatry Psychiatr Epidemiol. 2018;53(9):921-30.

3. Vargas HO, Nunes SOV, Barbosa DS, Vargas MM, Cestari A, Dodd S, et al. Castelli risk indexes 1 and 2 are higher in major depression but other characteristics of the metabolic syndrome are not specific to mood disorders. Life sciences. 2014;102(1):65-71.

4. Matta J, Hoertel N, Kesse-Guyot E, Plesz M, Wiernik E, Carette C, et al. Diet and physical activity in the association between depression and metabolic syndrome: Constances study. J Affect Disord. 2019:244:25-32.

5. Depression will be the second leading cause of disease by 2020. WHO. Available from: http://www.calgaryherald.com/health/Depression+will+ second+leading+cause+disease+2020/3640325/story.html.

6. Depression Now World's Second Leading Cause of Disability. Available from: https://www.medscape.com/viewarticle/813896.

7. East C, Willis BL, Barlow CE, Grannemann BD, FitzGerald SJ, DeFina LF, et al. Depressive symptoms and metabolic syndrome in preventive healthcare: the Cooper Center longitudinal study. Metabolic Syndrome Related Disorders. 2010;8(5):451-7.

8. Foley DL, Morley KI, Madden PA, Heath AC, Whitfield JB, Martin NG. Major depression and the metabolic syndrome. Twin Research Human Genetics. 2010;13(4):347-58

9. Hildrum B, Mykletun A, Midthjell K, Ismail K, Dahl A. No association of depression and anxiety with the metabolic syndrome: the Norwegian HUNT study. Acta Psychiatr Scand. 2009;120(1):14-22.

10. Vogelzangs N, Beekman AT, Dik MG, Bremmer MA, Comijs HC, Hoogendijk WJ, et al. Late-life depression, cortisol, and the metabolic syndrome. The American journal of geriatric psychiatry. 2009;17(8):716-21.

11. Yu S, Yang H, Guo X, Zheng L, Sun Y. Metabolic syndrome and depressive symptoms among rural Northeast general population in China. BMC Public Health. 2017;17(1):43

12. Chang $\mathrm{H}-\mathrm{C}$, Hsiao T-M, Lien M-H, Yeh C-J, Yang H-J. Metabolic syndrome and depression are not correlated: results from a community sample 
exploring the unique and common correlates for the two diseases. Neuropsychiatry. 2017;7(2):142-8.

13. Sekita A, Arima H, Ninomiya T, Ohara T, Doi Y, Hirakawa Y, et al. Elevated depressive symptoms in metabolic syndrome in a general population of Japanese men: a cross-sectional study. BMC Public Health. 2013;13(1):862.

14. Lim H, Nguyen T, Choue R, Wang Y. Sociodemographic disparities in the composition of metabolic syndrome components among adults in South Korea. Diabetes Care. 2012;35(10):2028-35.

15. Moher D, Liberati A, Tetzlaff J, Altman DG, Group P. Preferred reporting items for systematic reviews and meta-analyses: the PRISMA statement. PLoS med. 2009;6(7):e1000097.

16. Farrugia M, Kirsch A. Application of the Strengthening the Reporting of Observational Studies in Epidemiology (STROBE) statement to publications on endoscopic treatment for vesicoureteral reflux. J Pediatr Urol. 2017;13(3):320-5.

17. Luchini C, Stubbs B, Solmi M, Veronese N. Assessing the quality of studies in meta-analyses: Advantages and limitations of the Newcastle Ottawa Scale. World Journal of Meta-Analysis. 2017;5(4):80-4

18. Higgins J, Tompson S, Deeks J, Altman D. A Meta-Analysis on the Effectiveness of Smart-Learning. BMJ. 2003;327(1):p557-60.

19. Higgins J, Green S. Cochrane Handbook for Systematic Reviews of Interventions. Version 5.1.0 [updated March 2011]. [WWW Document]. Cochrane Collab. URL (www.cochrane-handbook.org). 2011.

20. Moreira FP, Jansen K, de Azevedo Cardoso T, Mondin TC, Vieira IS, da Silva Magalhaes PV, et al. Metabolic syndrome, depression and anhedonia among young adults. Psychiatry research. 2019;271:306-10.

21. Moreira FPJK, de Azevedo Cardoso T, Mondin TC, da Silva Magalhaes PV, Kapczinski F, de Mattos Souza LD, da Silva RA, Oses JP, Wiener CD. Metabolic syndrome in subjects with bipolar disorder and major depressive disorder in a current depressive episode: Population-based study: Metabolic syndrome in current depressive episode. J Psychiatr Res. 2017;92:119-23.

22. Ribeiro RP, Marziale MHP, Martins JT, Ribeiro PHV, Robazzi MLdCC, Dalmas JC. Prevalence of Metabolic Syndrome among nursing personnel and its association with occupational stress, anxiety and depression. Rev Latinoam Enferm. 2015;23(3):435-40.

23. Ko J-K, Han K-M, Shin C, Lee S-H, Han C, Kim Y-K, et al. Association of metabolic syndrome and its components with suicidal ideation and depression in adults: A nationally representative sample of the Korean population. J Affect Disord. 2019;249:319-26.

24. Kim Y, Kim H-Y. Association Between Depression and Metabolic Syndrome in Korean Adults: Data From the 2014 and 2016 Korea National Health and Nutrition Examination Survey. Asia Pacific Journal of Public Health. 2018; 31(1):18-29.

25. Ra JS, Kim HS. Sex-based association between depression and metabolic syndrome in Korean middle-aged and older adults. Osong public health research perspectives. 2017;8(2):130

26. Park SJ, Roh S, Hwang J, Kim HA, Kim S, Lee TK, et al. Association between depression and metabolic syndrome in korean women: Results from the korean national health and nutrition examination survey (2007-2013). J Affect Disord. 2016;205:393-9.

27. Rethorst C, Leonard D, Barlow C, Willis B, Trivedi M, DeFina L. Effects of depression, metabolic syndrome, and cardiorespiratory fitness on mortality: results from the Cooper Center longitudinal study. Psychological medicine. 2017;47(14):2414-20

28. Gurka MJ, Vishnu A, Okereke Ol, Musani S, Sims M, DeBoer MD. Depressive symptoms are associated with worsened severity of the metabolic syndrome in African American women independent of lifestyle factors: A consideration of mechanistic links from the Jackson heart study. Psychoneuroendocrinology. 2016;68:82-90.

29. Olvera RL, Williamson DE, Fisher-Hoch SP, Vatcheva KP, McCormick JB. Depression, obesity, and metabolic syndrome: Prevalence and risks of comorbidity in a population-based study of Mexican Americans. J Clin Psychiatry. 2015;76(10):e1300.

30. Goldbacher EM, Bromberger J, Matthews KA. Lifetime history of major depression predicts the development of the metabolic syndrome in middle-aged women. Psychosom Med. 2009;71(3):266.

31. Vaccarino V, McClure C, Johnson BD, Sheps DS, Bittner $V$, Rutledge $T$, et al. Depression, the metabolic syndrome and cardiovascular risk. Psychosom Med. 2008;70(1):40-8.

32. Chattopadhyay S, Bhattacharya K, Roy D, Chatterjee SS, Sadhukhan S. Comparative study of prevalence of metabolic syndrome in depressed patients attending a tertiary care centre in eastern india. J Evolution Med Dent Sci. 2018;7(17):2089-94.

33. Chan KL, Cathomas F, Russo SJ. Central and peripheral inflammation link metabolic syndrome and major depressive disorder. Physiology. 2019;34(2): 123-33.

34. Dregan A, Rayner L, Davis KA, Bakolis I, de la Torre JA, Das-Munshi J, et al. Associations between depression, arterial stiffness, and metabolic syndrome among adults in the UK Biobank Population Study: a mediation analysis. JAMA psychiatry. 2020.

35. Viinamäki H, Heiskanen $T$, Lehto $S$, Niskanen L, Koivumaa-Honkanen $H$, Tolmunen T, et al. Association of depressive symptoms and metabolic syndrome in men. Acta Psychiatr Scand. 2009;120(1):23-9.

36. Toker S, Shirom A, Melamed S. Depression and the metabolic syndrome: gender-dependent associations. Depress Anxiety. 2008;25(8):661-9.

37. Kinder LS, Carnethon MR, Palaniappan LP, King AC, Fortmann SP. Depression and the metabolic syndrome in young adults: findings from the Third National Health and Nutrition Examination Survey. Psychosom Med. 2004;66(3):316-22

38. Takeuchi T, Nakao M, Kachi Y, Yano E. Association of metabolic syndrome with atypical features of depression in J apanese people. J Neuropsychiatry Clin Neurosci. 2013:67(7):532-9.

39. Vanhala M, Jokelainen J, Keinänen-Kiukaanniemi S, Kumpusalo E, Koponen H. Depressive symptoms predispose females to metabolic syndrome: a 7year follow-up study. Acta Psychiatr Scand. 2009;119(2):137-42.

40. Agarwal A, Agarwal M, Garg K, Dalal PK, Trivedi JK, Srivastava J. Metabolic syndrome and central obesity in depression: A cross-sectional study. Indian journal of psychiatry. 2016;58(3):281.

41. Ahola AJ, Thorn LM, Saraheimo M, Forsblom C, Groop P-H, Group FS. Depression is associated with the metabolic syndrome among patients with type 1 diabetes. Annals of medicine. 2010;42(7):495-501.

42. Akbaraly TN, Kivimäki M, Brunner EJ, Chandola T, Marmot MG, SinghManoux A, et al. Association between metabolic syndrome and depressive symptoms in middle-aged adults: results from the Whitehall II study. Diabetes Care. 2009;32(3):499-504.

43. Bakhtiari A, Hashemi M, Hosseini SR, Omidvar S, Bijani A, Khairkhah F. The Relationship between Depression and Metabolic Syndrome in the Elderly Population: The Cohort Aging Study. Iranian journal of psychiatry. 2018; 13(4):230-8

44. Mattei G, Padula MS, Rioli G, Arginelli L, Bursi R, Bursi S, et al. Metabolic syndrome, anxiety and depression in a sample of Italian primary care patients. J Nerv Ment Dis. 2018;206(5):316-24.

45. Cardenas V, Mausbach BT, Sommerfeld D, Jimenez D, von Känel R, Ho JS, et al. Depression is associated with increased risk for metabolic syndrome in Latinos with type 2 diabetes. The American Journal of Geriatric Psychiatry. 2017:25(6):646-53.

46. Kahl KG, Schweiger U, Correll C, Müller C, Busch ML, Bauer M, et al. Depression, anxiety disorders, and metabolic syndrome in a population at risk for type 2 diabetes mellitus. Brain behavior. 2015;5(3):e00306.

47. Butnoriene J, Bunevicius A, Norkus A, Bunevicius R. Depression but not anxiety is associated with metabolic syndrome in primary care based community sample. Psychoneuroendocrinology. 2014;40:269-76.

48. Demirci H, Cinar Y, Bilgel N. Metabolic syndrome and depressive symptoms in a primary health care setting in Turkey. Klinik Psikofarmakoloji BülteniBulletin of Clinical Psychopharmacology. 2011;21(1):49-57.

49. Miettola J, Niskanen LK, Viinamäki H, Kumpusalo E. Metabolic syndrome is associated with self-perceived depression. Scand J Prim Health Care. 2008; 26(4):203-10

50. Dunbar JA, Reddy P, Davis-Lameloise N, Philpot B, Laatikainen T, Kilkkinen A, et al. Depression: an important comorbidity with metabolic syndrome in a general population. Diabetes Care. 2008;31(12):2368-73.

51. Vogelzangs N, Suthers K, Ferrucci L, Simonsick EM, Ble A, Schrager M, et al. Hypercortisolemic depression is associated with the metabolic syndrome in late-life. Psychoneuroendocrinology. 2007;32(2):151-9.

52. Skilton MR, Moulin P, Terra J-L, Bonnet F. Associations between anxiety, depression, and the metabolic syndrome. Biol Psychiatry. 2007;62(11):1251-7.

53. Virtanen $M$, Ferrie JE, Akbaraly $T$, Tabak $A$, Jokela $M$, Ebmeier KP, et al. Metabolic Syndrome and Symptom Resolution in Depression: A 5-Year Follow-Up of Older Adults. J Clin Psychiatry. 2017;78(1):e1-7.

54. Akbaraly TN, Ancelin M-L, Jaussent I, Ritchie C, Barberger-Gateau P, Dufouil $C$, et al. Metabolic syndrome and onset of depressive symptoms in the elderly: findings from the three-city study. Diabetes Care. 2011;34(4):904-9. 
55. Räikkönen K, Matthews KA, Kuller LH. Depressive symptoms and stressful life events predict metabolic syndrome among middle-aged women: a comparison of World Health Organization, Adult Treatment Panel III, and International Diabetes Foundation definitions. Diabetes Care. 2007;30(4):872-7.

56. Gil K, Radziwillowicz P, Zdrojewski T, Chwojnicki K, Piwonski J, IgnaszewskaWyrzykowska A, et al. Relationship between the prevalence of depressive symptoms and metabolic syndrome. Results of the SOPKARD Project. Kardiologia Polska. 2006;64(5):464.

57. Herva A, Räsänen P, Miettunen J, Timonen M, Läksy K, Veijola J, et al. Cooccurrence of metabolic syndrome with depression and anxiety in young adults: the Northern Finland 1966 Birth Cohort Study. Psychosom Med. 2006;68(2):213-6.

58. Gheshlagh RG, Parizad N, Sayehmiri K. The relationship between depression and metabolic syndrome: systematic review and meta-analysis study. Iranian Red Crescent Medical Journal. 2016;18(6):1-6.

59. Pan A, Keum N, Okereke Ol, Sun Q, Kivimaki M, Rubin RR, et al. Bidirectional association between depression and metabolic syndrome: a systematic review and meta-analysis of epidemiological studies. Diabetes Care. 2012; 35(5):1171-80

60. Vancampfort D, Correll CU, Wampers M, Sienaert P, Mitchell A, De Herdt A, et al. Metabolic syndrome and metabolic abnormalities in patients with major depressive disorder: a meta-analysis of prevalences and moderating variables. Psychological medicine. 2014;44(10):2017-28.

61. Banerjee S, Varma RP. Factors affecting non-adherence among patients diagnosed with unipolar depression in a psychiatric department of a tertiary hospital in Kolkata, India. Depression research and treatment. 2013;2013.

62. DiMatteo MR, Lepper HS, Croghan TW. Depression is a risk factor for noncompliance with medical treatment: meta-analysis of the effects of anxiety and depression on patient adherence. Arch Intern Med. 2000; 160(14):2101-7.

63. van Reedt Dortland AK, Giltay EJ, Van Veen T, Zitman FG, Penninx BW. Metabolic syndrome abnormalities are associated with severity of anxiety and depression and with tricyclic antidepressant use. Acta Psychiatr Scand. 2010:122(1):30-9.

64. Grosso G, Micek A, Castellano S, Pajak A. Galvano FJMn, research f. Coffee, tea, caffeine and risk of depression: A systematic review and dose-response meta-analysis of observational studies. 2016;60(1):223-34.

65. Grosso G, Micek A, Marventano S, Castellano S, Mistretta A, Pajak A, et al. Dietary n-3 PUFA, fish consumption and depression: A systematic review and meta-analysis of observational studies. 2016;205:269-81.

66. Li F, Liu X, Zhang D. Fish consumption and risk of depression: a metaanalysis. 2016;70(3):299-304.

67. Le Port A, Gueguen A, Kesse-Guyot E, Melchior M, Lemogne C, Nabi H, et al. Association between dietary patterns and depressive symptoms over time: a 10-year follow-up study of the GAZEL cohort. PloS one. 2012;7:12.

68. Huang Q, Liu H, Suzuki K, Ma S, Liu CJA. Linking what we eat to our mood: A review of diet, dietary antioxidants, and depression. 2019:8(9):376.

69. Allison MA, Budoff MJ, Wong ND, Blumenthal RS, Schreiner PJ, Criqui MH. Prevalence of and risk factors for subclinical cardiovascular disease in selected US Hispanic ethnic groups: the Multi-Ethnic Study of Atherosclerosis. Am J Epidemiol. 2008;167(8):962-9.

70. Raikkonen K, Matthews KA, Kuller LH. The relationship between psychological risk attributes and the metabolic syndrome in healthy women: antecedent or consequence? Metabolism-Clinical Experimental. 2002;51(12):1573-7.

71. Persaud D. Gender Differences And Fast Food Preferences Among US College Students. University of Central Florida 2013.

72. Men. and Women Don't Have the Same Fast Food Habits - See the Key Difference. Avaliable from: https://www.eatthis.com/men-womenfast-food-habits/.

\section{Publisher's Note}

Springer Nature remains neutral with regard to jurisdictional claims in published maps and institutional affiliations.

\section{Ready to submit your research? Choose BMC and benefit from:}

- fast, convenient online submission

- thorough peer review by experienced researchers in your field

- rapid publication on acceptance

- support for research data, including large and complex data types

- gold Open Access which fosters wider collaboration and increased citations

- maximum visibility for your research: over $100 \mathrm{M}$ website views per year

At $\mathrm{BMC}$, research is always in progress.

Learn more biomedcentral.com/submissions 